\title{
Synthesis of macrocyclic cyclophane-based unusual $\alpha$-amino acid derivatives
}

\author{
Sambasivarao Kotha* and Somnath Halder \\ Department of Chemistry, Indian Institute of Technology-Bombay, Powai, \\ Mumbai 400 076, India \\ E-mail: srk@chem.iitb.ac.in
}

Dedicated to Dr. A. V. Rama Rao on the occasion of his $70^{\text {th }}$ birthday

(received 29 Jun 04; accepted 22 Sep 04; published on the web 02 Oct 04)

\begin{abstract}
Alkylation of ethyl isocyanoacetate with 1,2-bis(4-bromomethylphenyl)ethane under phase transfer catalysis (PTC) as well as phosphazene base [2-tert-butylimino-2-diethylamino-1,3dimethylperhydro-1,3,2-diazaphosphorine (BEMP)] conditions gave the macrocyclic cyclophane based unusual $\alpha$-amino acid derivatives.
\end{abstract}

Keywords: Amino acids, cyclophanes, macrocycles, alkylation

\section{Introduction}

$\mathrm{C}^{\alpha \alpha}$-Disubstituted glycines have been proved to be useful tools in the designing of small "drug like" molecules. ${ }^{1}$ In addition, $C^{\alpha \alpha}$-dialkylated $\alpha$-amino acid (AAA) residue such as Aib $(\alpha$ aminoisobutyric acid) $\mathbf{1}$ is commonly being used as an important structural element for stabilizing helical conformations. In this regard, we are interested in preparing unusual AAA derivatives containing cyclophane moiety (e.g., 3), which is a hybrid of Aib (1) and [3,2]paracyclophane unit $(\mathbf{2})^{2}$ (Figure 1). Homo-chiral amino acid, not AAA based on $[2,2]$ paracyclophane unit has been prepared $^{3}$ with the aim of introducing in peptide chain to modify the topology and lipophilicity.

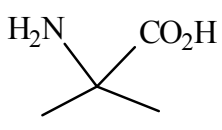

1

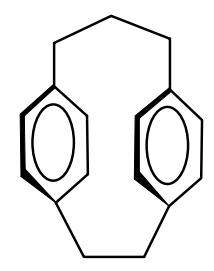

2

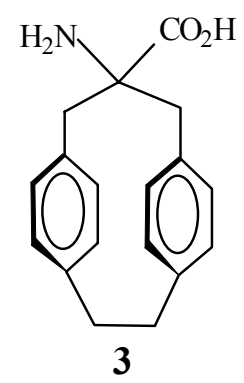

Figure 1 


\section{Strategy}

Our synthetic approach to cyclophane-based AAA 3 involves two different routes. The first route (path a) involves ring-closing metathesis (RCM) reaction as a key step. ${ }^{4}$ Alternatively, the key $\mathrm{C}-\mathrm{C}$ bond can be formed by alkylation of a suitable glycine equivalent with appropriately substituted aromatic derivative (path b) (Scheme 1). For example, alkylation of $\alpha, \alpha^{\prime}$-dibromo- $p$ xylene with glycine equivalent can deliver the required para cyclophane ring system. This approach is reminiscent of Sasaki's work. ${ }^{\mathbf{5}}$ Although, the strategy shown in Scheme 1, has not given the target compound but it delivered interesting dimers, based on cyclophane system.

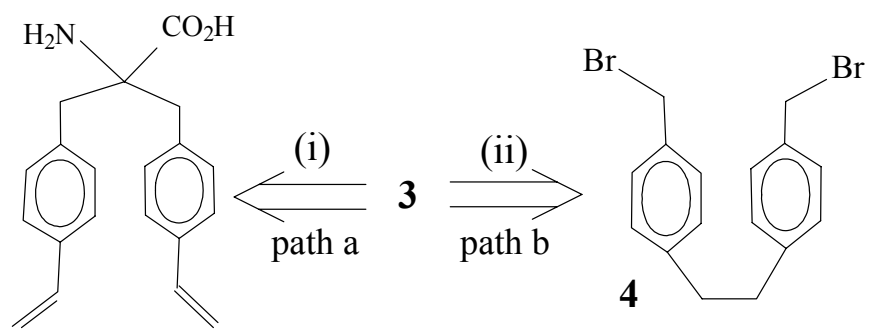

Scheme 1. (i) RCM, $\mathrm{H}_{2} / \mathrm{Pd}-\mathrm{C}$ (ii) Glycine equivalent.

\section{Results and Discussion}

To realize the first strategy (path a), ethyl isocyanoacetate ${ }^{6}$ was treated with 4-vinylbenzyl chloride (5) in presence of $\mathrm{NaH} / \mathrm{DMSO}$ at $0{ }^{\circ} \mathrm{C}$ to deliver dialkylated product 6 in $90 \%$ yield (Scheme 2). Compound 6 was converted to $\mathrm{N}$-formyl and $\mathrm{N}$-acetyl derivative $\mathbf{7 a}$ and $\mathbf{7 b}$ respectively. The RCM reaction of compounds $\mathbf{7 a}$ and $\mathbf{7 b}$ in presence of Grubbs' catalyst [bis(tricyclohexyl-phosphine)benzylidine ruthenium (IV) dichloride] were found to be unsuccessful. ${ }^{7}$ Later on, an alternate strategy (path $\mathbf{b}$ ) was attempted.

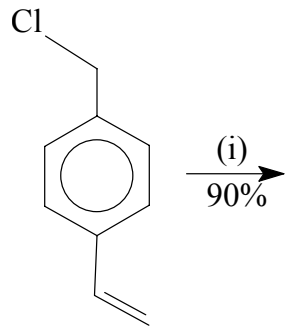

5

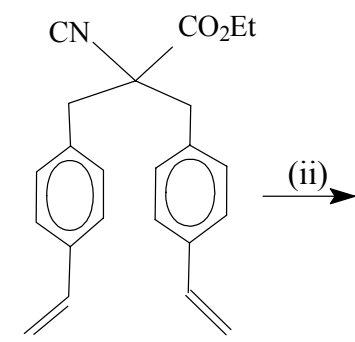

6

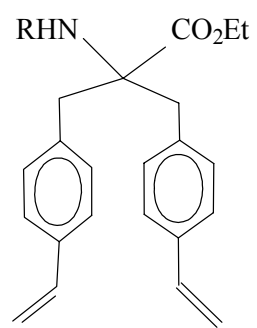

7a. $\mathrm{R}=\mathrm{CHO}(98 \%)$

7b. $\mathrm{R}=\mathrm{Ac}(81 \%)$

Scheme 2. (i) $\mathrm{CNCH}_{2} \mathrm{CO}_{2} \mathrm{ET}, \mathrm{NaH}, \mathrm{DMSO}$, ether, RT.

Alkylation of Schiff-base ${ }^{\mathbf{8}}$ with $\mathbf{4}^{\mathbf{9}}$ in presence of $\mathrm{KOH} / \mathrm{TBAB}$ (tetra- $n$-butylammonium bromide) in acetonitrile at $0{ }^{\circ} \mathrm{C}$ followed by hydrolysis and subsequent protection with acetic anhydride gave $\mathrm{N}$-acetyl derivatives 8 (8\%) and 9 (7\%) (Scheme 3) instead of the expected 
cyclophane derivative related to 3. Again alkylation in presence of micelles such as cetyltrimethylammonium bromide $(\mathrm{CTAB})$ was tried so that, the favorable entropy conditions may aid the formation of the required cyclophane derivative. ${ }^{\mathbf{1 0}}$

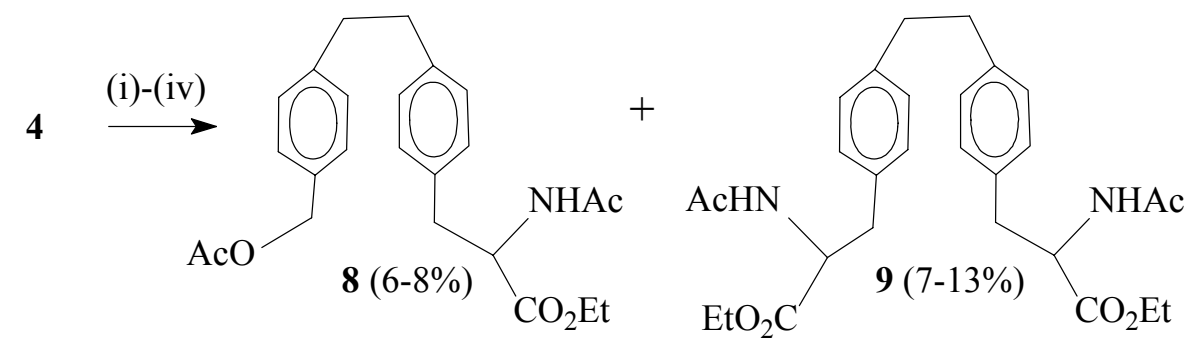

Sheme 3. (i) Schiff-base (ii) $\mathrm{KOH}, \mathrm{TRAB}$, or micelle (CTAB), $\mathrm{CH}_{3} \mathrm{CN}$ (iii) $\mathrm{H}^{+}$, ether, RT (iv) $\mathrm{Ac}_{2} \mathrm{O}, \mathrm{DMAP}, \mathrm{CH}_{2} \mathrm{Cl}_{2}$, RT.

Under these conditions, reaction of $\mathbf{4}$ with Schiff-base followed by hydrolysis and acetylation also gave 8 (6\%) and 9 (13\%) (yields refer to overall yield starting from bromide 4). Since bisarmed AAAs are important structural motif in several antibiotics, the methodology found here may find useful application in medicinal chemistry. ${ }^{11}$

Since ethyl isocyanoacetate has been found to be a useful glycine equivalent, we tried to use this synthon for the synthesis of cyclophane-based AAA derivatives. Hence, the dibromide 4 was treated with ethyl isocyanoacetate under PTC conditions which has given the alkylated product 10 (mp 190-192 ${ }^{\circ} \mathrm{C}$ ) in 3.5\% yield (Scheme 4).
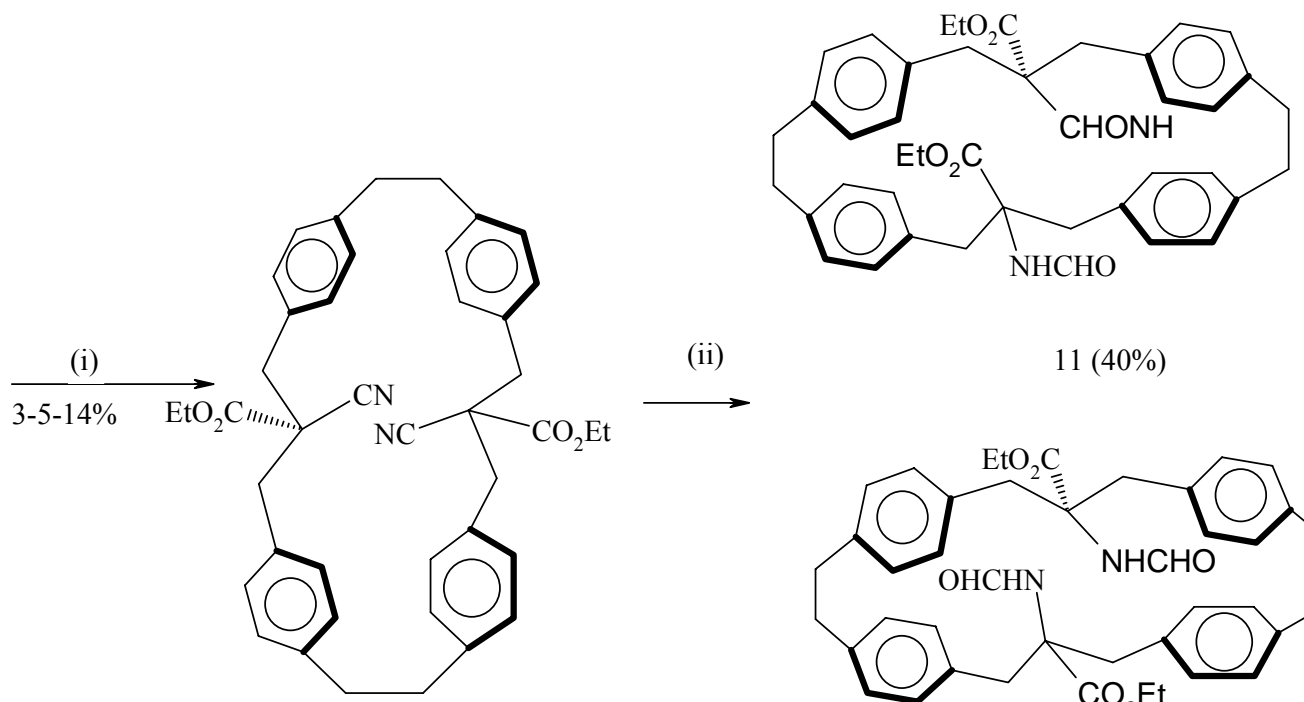

(ii)

$11(40 \%)$

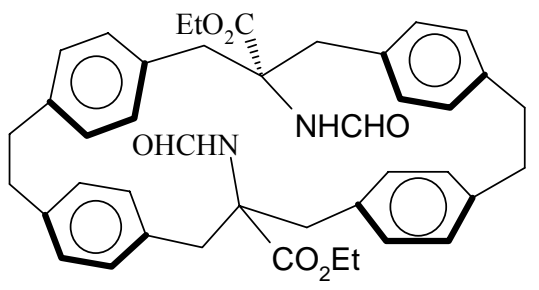

10

$12(48 \%)$ 
Scheme 4. (i) $\mathrm{CNCH}_{2} \mathrm{CO}_{2} \mathrm{Et}, \mathrm{K}_{2} \mathrm{CO}_{3}, \mathrm{CH}_{3} \mathrm{CN}, \Delta$ or BEMP, $\mathrm{CH}_{3} \mathrm{CN}, 0{ }^{\circ} \mathrm{C}$ (ii) $\mathrm{H}^{+}$, ehter $0{ }^{\circ} \mathrm{C}$ - $\mathrm{RT}$.

It is reported that the dibromide 4 can form radical intermediates under thermal or photochemical reaction conditions that undergo a disproportion/polymerization reaction. ${ }^{\mathbf{2}}$ The low yield of the coupling product $\mathbf{1 0}$ can be explained due to possibility of this unwanted side reactions. To confirm this, dibromide 4 was treated in absence of ethyl isocyanoacetate under PTC conditions and obtained poor recovery (28\%) of dibromide 4.

Several other conditions have been tried to improve the yield of $\mathbf{1 0}$, for instance NaH/DMSO conditions gave much lower yield compare to PTC conditions. It is known that the phosphazene bases improve the yields of coupling product when sensitive substrates are involved by minimizing the unwanted side-reactions. In this respect, 2-tert-butylimino-2-diethylamino-1,3dimethylperhydro-1,3,2-diazaphosphorine (BEMP) ${ }^{\mathbf{1 3}}$ was used as a base in acetonitrile at $0{ }^{\circ} \mathrm{C}$. The coupling product $\mathbf{1 0}$ was isolated in 14\% yield (4-fold increase as compared to PTC conditions) as a mixture of isomeric products. Hydrolysis of coupling product $\mathbf{1 0}$ in presence of $\mathrm{HCl}$ /diethyl ether at RT gave the $\mathrm{N}$-formyl derivative 11 (mp. $220{ }^{\circ} \mathrm{C}$ decomp.) and another isomeric product 12 (mp $210{ }^{\circ} \mathrm{C}$ decomp.) in $40 \%$ and $48 \%$ isolated yields respectively. The structure of 12 has been established by X-ray studies. ${ }^{14}$ The structure of 11 could not be established by crystallographic analysis because suitable crystals could not obtained. After successful synthesis of macrocyclic cyclophane-based AAAs 11 and $\mathbf{1 2}$ under PTC and BEMP conditions, we decided to extened this methodolgy to the corresponding benzo analogues such as 14 starting from dibromide 13 (Eq 1).

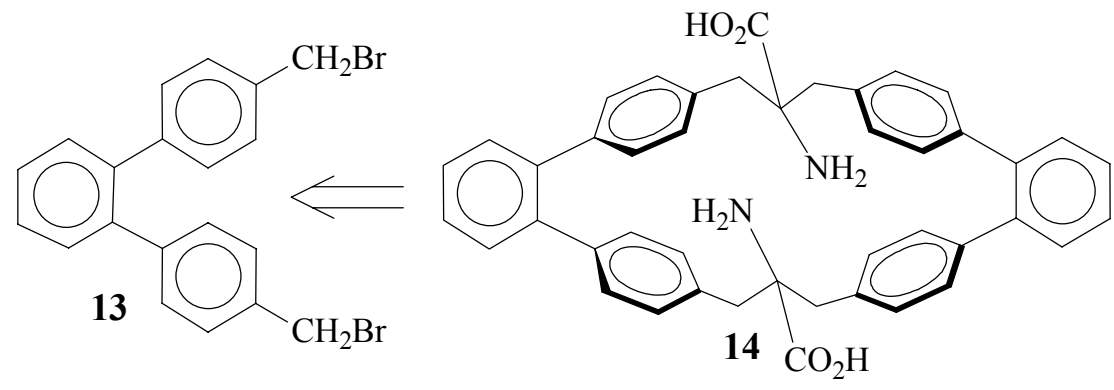

Towards this goal, the precursor of dibromide 13 was prepared by an alternative method. Thus, the Suzuki coupling reaction ${ }^{15}$ of 1,2-dibromobenzene with $p$-methylphenylboronic acid in presence of $\mathrm{Pd}\left(\mathrm{PPh}_{3}\right)_{4}$ catalyst gave the cross-coupling product 15 in $86 \%$ yield. Benzylic bromination of $\mathbf{1 5}$ was performed according to the known procedure ${ }^{\mathbf{1 6}}$ (Scheme 5). Alkylation of ethyl isocyanoacetate with dibromide 13 under BEMP base conditions gave cyclophane derivative $16(25 \%)\left(\mathrm{mp} 245{ }^{\circ} \mathrm{C}\right.$, decomp). The spectral data of 16 indicates a mixture of isomeric products. With the hope of isolating the individual isomer after hydrolysis reaction, compound 16 was hydrolized in presence of $\mathrm{HCl}$ diethyl ether and product $\mathbf{1 7}$ was obtained. The spectral data of $\mathbf{1 7}$ also shows a mixture of isomers. TLC analysis under different solvent system indicated only one spot and unfortunately we were not able to separate them by silica gel (or 
alumina) column chromatography. The high-resolution mass spectrum of $\mathbf{1 7}$ shows a peak at $(\mathrm{M}+1) 771.34243$ corresponding to molecular formulation $\left(\mathrm{C}_{50} \mathrm{H}_{46} \mathrm{~N}_{2} \mathrm{O}_{6}\right)$.

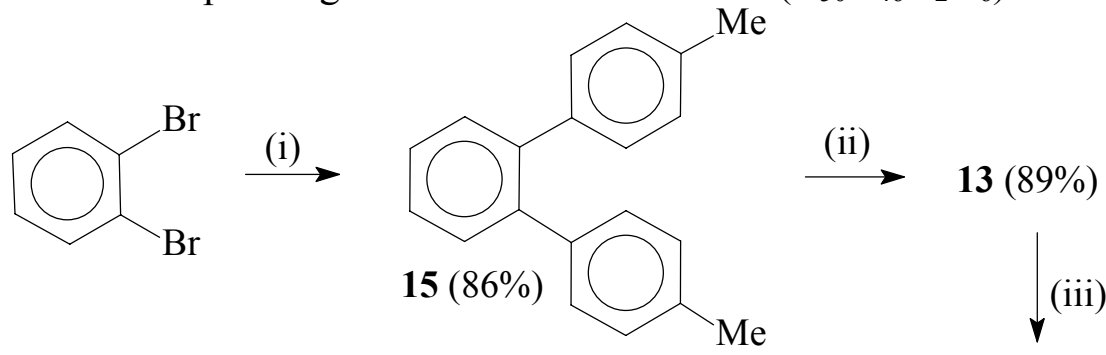

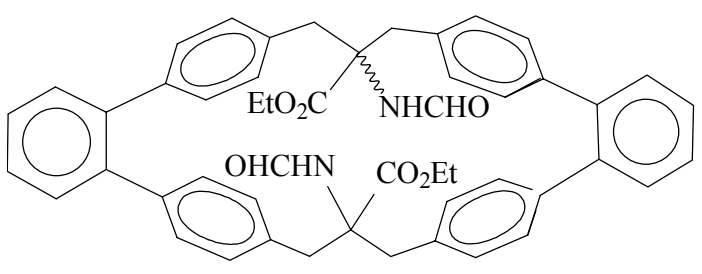

$17(62 \%)$

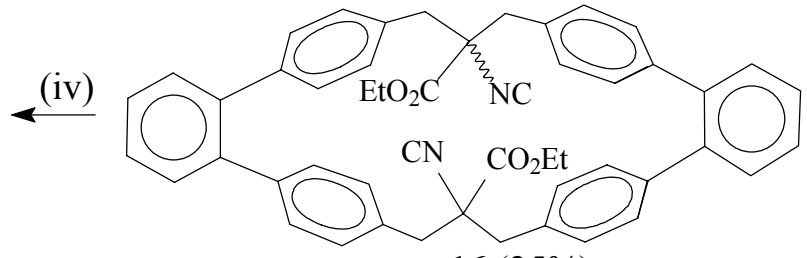

$16(25 \%)$

Scheme 5. (i) $p$ - $\mathrm{MePhB}(\mathrm{OH})_{2}, \mathrm{Pd}(0), \mathrm{Na}_{2} \mathrm{CO}_{3}$, THF, toluene, $\mathrm{H}_{2} \mathrm{O}$ (ii) $\mathrm{NBS}, \mathrm{AIBN} \mathrm{CCl}_{4}$ (iii) BEMP, $\mathrm{CH}_{3} \mathrm{CN}, 0{ }^{\circ} \mathrm{C}$ (iv) $\mathrm{HCl}$, Ether.

\section{Conclusions}

We were able to synthesize macrocyclic cyclophane-based AAA derivatives (racemic) 11, 12 and 17 by using ethyl isocyanoacetate as a glycine equivalent. Using phosphazene base (BEMP) the yields of the coupling step has been improved considerably. Use of phosphazene bases may also find applications in synthesis of cyclophane dertivatives.

\section{Experimental Section}

General Procedures. Dry diethyl ether, benzene and toluene have been obtained by distillation over sodium benzophenone ketyl. Chloroform, dichloromethane, carbon tetrachloride and acetonitrile were distilled over $\mathrm{P}_{2} \mathrm{O}_{5}$. BEMP and 4-vinylbenzyl chloride were purchased from Aldrich Chemical Co, Milwauke, WI, U. S. A. Grubbs' catalyst was purchased from Strem Chemical Co, Newburyport, MA. U. S. A. ${ }^{1} \mathrm{H}$ NMR spectra were recorded on Bruker $300 \mathrm{MHz}$ or Hitachi FT-60 MHz Instrument. ${ }^{13} \mathrm{C}$ NMR spectra were recorded on Bruker $75.4 \mathrm{MHz}$ instrument. High resolution mass spectra were obtained on JEOL JMS-DX 303 GC-MS instrument. The FAB mass spectra were obtained on JEOL SX 102/DA-6000 mass spectrometer. 
UV Spectra were obtained on Shimadzu UV-2100 instrument. Room temperature IR spectra were obtained on Nicolet Impact-400 FT IR spectrometer.

Synthesis of compound (6). To a stirred solution of 4-vinylbenzyl chloride (5) (1 g, 6.55 mmol), ethyl isocyanoacetate $(370 \mathrm{mg}, 3.27 \mathrm{mmol})$ and dry DMSO (6.82 g, $87.2 \mathrm{mmol})$ in dry diethyl ether $(25 \mathrm{ml})$ was slowly added a slurry of (washed with dry petroleum ether) $60 \% \mathrm{NaH}$ (262 $\mathrm{mg}, 6.55 \mathrm{mmol})$ in dry diethyl ether $(10 \mathrm{ml})$ at $15{ }^{\circ} \mathrm{C}$. After $4 \mathrm{~h}$ at RT the reaction mixture was quenched with water $(2 \mathrm{ml})$ and extracted with diethyl ether $(25 \mathrm{ml} \times 3)$, washed with water $(20$ $\mathrm{ml})$, brine $(10 \mathrm{ml})$, dried over $\mathrm{MgSO}_{4}$ and evaporated. The residue was chromatographed on silica gel in $1 \%$ ethyl acetate/petroleum ether to lead to dialkylated product $6(1.02 \mathrm{~g}, 90 \%)$ as a colorless liquid. $R_{f}$ (ethyl acetate : petroleum ether 1:9): 0.50; $\mathrm{UV}\left(\mathrm{CHCl}_{3}\right): \lambda_{\max }\left(\varepsilon \mathrm{M}^{-1} \mathrm{~cm}^{-1}\right)$ $294.5\left(1.42 \times 10^{3}\right), 257.0\left(3.08 \times 10^{4}\right) \mathrm{nm}$; IR $(\mathrm{KBr}) v_{\max }: 2137(\mathrm{NC}), 1742($ ester $\mathrm{C}=\mathrm{O}) \mathrm{cm}^{-1} ;{ }^{1} \mathrm{H}$ NMR (300 MHz, $\left.\mathrm{CDCl}_{3}\right): \delta 1.06\left(\mathrm{t}, \mathrm{J}=7.0 \mathrm{~Hz}, 3 \mathrm{H}\right.$, ester $\left.\mathrm{CH}_{3}\right), 3.05(1 / 2 \mathrm{ABq}, \mathrm{J}=13.5 \mathrm{~Hz}, 2 \mathrm{H}$, diastereotopic-H), 3.33 (1/2 ABq, J=13.5 Hz, 2H, diastereotopic-H), 4.05 (q. J=7.0 Hz, 2H, ester $\left.\mathrm{OCH}_{2}\right), 5.24$ (d, J=10.9 Hz, 2H, alkene-H), 5.73 (d, J=17.5 Hz, 2H, alkene-H), 6.70 (dd, $\mathrm{J}_{1}=17.5$, $\mathrm{J}_{2}=10.9 \mathrm{~Hz}, 2 \mathrm{H}$, alkene-H), $7.25(\mathrm{~d}, \mathrm{~J}=8.0 \mathrm{~Hz}, 4 \mathrm{H}, \mathrm{Ar}-\mathrm{H}), 7.38(\mathrm{~d}, \mathrm{~J}=8.0 \mathrm{~Hz}, 4 \mathrm{H}, \mathrm{Ar}-\mathrm{H}) ;{ }^{13} \mathrm{C}$ NMR (75.4 MHz, $\left.\mathrm{CDCl}_{3}\right): \delta 13.8,44.5,62.6,70.1,114.1,126.2,130.4,133.0,136.3,137.1$, 161.2, 167.8; Mass: m/z $345\left(\mathrm{M}^{+}\right)$; HRMS: m/z (EI) for $\mathrm{C}_{23} \mathrm{H}_{23} \mathrm{NO}_{2}$ Calcd: 345.1728; Found: 345.1723 .

Synthesis of compound (7a). To a stirred solution of isonitrile derivative 6 (290 $\mathrm{mg}, 0.84 \mathrm{mmol}$ ) in diethyl ether $(10 \mathrm{ml})$ was added concd $\mathrm{HCl}(8$ drops $)$ at $0{ }^{\circ} \mathrm{C}$. After $1 \mathrm{~h}$ at $\mathrm{RT}$, water $(2 \mathrm{ml})$ was added, then extracted with diethyl ether $(20 \mathrm{ml} \times 3)$, washed with water $(20 \mathrm{ml})$, brine $(20 \mathrm{ml})$, dried over $\mathrm{MgSO}_{4}$ and evaporated. The residue gave pure $\mathrm{N}$-formyl derivative 7a with out any purification (300 mg, 98\%) as a white solid. Mp: 106-108 ${ }^{\circ} \mathrm{C} ; R_{f}$ (ethyl acetate : petroleum ether 1:9): 0.40; UV $\left(\mathrm{CHCl}_{3}\right): \lambda_{\max }\left(\varepsilon \mathrm{M}^{-1} \mathrm{~cm}^{-1}\right) 257.0\left(3.40 \times 10^{4}\right) \mathrm{nm}$; IR $(\mathrm{KBr}) v_{\max }: 3316(\mathrm{NH}), 1740$ $($ ester $\mathrm{C}=\mathrm{O}), 1667(\mathrm{NHC}=\mathrm{O}) \mathrm{cm}^{-1} ;{ }^{1} \mathrm{H}$ NMR $\left(300 \mathrm{MHz}, \mathrm{CDCl}_{3}\right): \delta 1.37(\mathrm{t}, \mathrm{J}=7.1 \mathrm{~Hz}, 3 \mathrm{H}$, ester $\left.\mathrm{CH}_{3}\right), 3.28(1 / 2 \mathrm{ABq}, \mathrm{J}=13.5 \mathrm{~Hz}, 2 \mathrm{H}$, diastereotopic-H), $3.95(1 / 2 \mathrm{ABq}, \mathrm{J}=13.5 \mathrm{~Hz}, 2 \mathrm{H}$, diastereotopic-H), 4.23 (q. J=7.1 Hz, 2H, ester $\mathrm{OCH}_{2}$ ), 5.22 (d, J=10.9 Hz, 2H, alkene-H), 5.69 $\left(\mathrm{d}, \mathrm{J}=17.5 \mathrm{~Hz}, 2 \mathrm{H}\right.$, alkene-H), $6.20(\mathrm{~s}, 1 \mathrm{H}, \mathrm{NH}), 6.65$ (dd, $\mathrm{J}_{1}=17.5 \mathrm{~Hz}, \mathrm{~J}_{2}=10.9 \mathrm{~Hz}, 2 \mathrm{H}$, alkeneH), 7.06 (d, J=8.0 Hz, 4H, Ar-H), 7.28 (d, J=8.0 Hz, 4H, Ar-H), 8.18 (d, J=1.8 Hz,. 1H, CHO); ${ }^{13} \mathrm{C}$ NMR (75.4 MHz, $\mathrm{CDCl}_{3}$ ): $\delta$ 14.4, 41.0, 62.5, 67.6, 113.9, 126.3, 130.0 (2C?), 135.7, 136.6, 161.0, 172.0; Mass: m/z 318 (M-CH3 $\mathrm{NO}$ ); HRMS: m/z (positive ion FAB) for $\mathrm{C}_{23} \mathrm{H}_{25} \mathrm{NO}_{3}$ Calcd: $(\mathrm{M}+\mathrm{H})$ 364.1912; Found: 364.1904.

Synthesis of compound (7b). To a stirred solution of isonitrile derivative 6 (180 mg, 0.52 $\mathrm{mmol})$ in absolute ethanol $(5 \mathrm{ml})$ was added concd $\mathrm{HCl}(5 \mathrm{drops})$ at $0{ }^{\circ} \mathrm{C}$. After 30 minutes at RT, solvent was removed under reduced pressure, water $(5 \mathrm{ml})$ was added, extracted with diethyl ether $(20 \mathrm{ml})$ and ether layer was discarded to remove organic residues. The aqueous layer was basified with $\mathrm{NH}_{3}$ solution to $\mathrm{pH} \sim 10$, extracted with ethyl acetate $(20 \mathrm{ml} \times 3)$, dried over $\mathrm{MgSO}_{4}$ and evaporated. The residue gave pure amino ester derivative $(18 \mathrm{mg}, 10 \%)$ as a thick liquid which was directly acetylated with acetic anhydride in the next step. 
To a solution of amino ester $(17 \mathrm{mg}, 0.05 \mathrm{mmol})$ in dry dichloromethane $(2 \mathrm{ml})$ were added acetic anhydride (2 drops) and a pinch of DMAP. After $24 \mathrm{~h}$ at RT, water $(5 \mathrm{ml})$ was added, extracted with dichloromethane $(15 \mathrm{ml} \times 3)$, washed with water $(15 \mathrm{ml})$, brine $(15 \mathrm{ml})$, dried over $\mathrm{MgSO}_{4}$ and evaporated. The residue was chromatographed on silica gel in $5 \%$ ethyl acetate/petroleum ether to lead to acetylated product $7 \mathbf{b}(15.5 \mathrm{mg}, 81 \%)$ as a semi solid. $R_{f}$ (ethyl acetate : petroleum ether 1:4): 0.57; $\mathrm{UV}\left(\mathrm{CHCl}_{3}\right): \lambda_{\max }\left(\varepsilon \mathrm{M}^{-1} \mathrm{~cm}^{-1}\right) 257.0\left(3.18 \times 10^{4}\right) \mathrm{nm}$; IR $(\mathrm{KBr}) v_{\max }: 3408(\mathrm{NH}), 1734($ ester $\mathrm{C}=\mathrm{O}), 1673(\mathrm{NHC}=\mathrm{O}) \mathrm{cm}^{-1} ;{ }^{1} \mathrm{H} \mathrm{NMR}\left(300 \mathrm{MHz}, \mathrm{CDCl}_{3}\right): \delta$ $1.40\left(\mathrm{t}, \mathrm{J}=7.1 \mathrm{~Hz}, 3 \mathrm{H}\right.$, ester $\left.\mathrm{CH}_{3}\right), 1.95\left(\mathrm{~s}, 3 \mathrm{H}, \mathrm{NCOCH}_{3}\right), 3.20(1 / 2 \mathrm{ABq}, \mathrm{J}=13.4 \mathrm{~Hz}, 2 \mathrm{H}$, diastereotopic-H), 4.01 (1/2 ABq, J=13.4 Hz, 2H, diastereotopic-H), 4.21 (q. J=7.1 Hz, 2H, ester $\left.\mathrm{OCH}_{2}\right), 5.20(\mathrm{~d}, \mathrm{~J}=10.9 \mathrm{~Hz}, 2 \mathrm{H}$, alkene-H), 5.44 (d, J=17.5 Hz, 2H, alkene-H), 6.10 (s, 1H, NH), $6.66\left(\mathrm{dd}, \mathrm{J}_{1}=17.5 \mathrm{~Hz}, \mathrm{~J}_{2}=10.9 \mathrm{~Hz}, 2 \mathrm{H}\right.$, alkene-H), 7.03 (d, J=8.2 Hz, 4H, Ar-H), 7.28 (d, J=8.2 $\mathrm{Hz}, 4 \mathrm{H}, \mathrm{Ar}-\mathrm{H}$ ); HRMS: m/z (positive ion FAB) for $\mathrm{C}_{24} \mathrm{H}_{27} \mathrm{NO}_{3}$ Calcd: $(\mathrm{M}+\mathrm{H}) 378.2069$; Found 378.2060 .

Synthesis of compound (8) and (9). A solution of dibromide 4 (200 mg, $0.54 \mathrm{mmol}$ ), N(diphenylmethylene)glycine ethyl ester (Schiff-base) $(175 \mathrm{mg}, 0.65 \mathrm{mmol})$ in dry acetonitrile (5 $\mathrm{ml}$ ) was added drop-wise to a solution of $\mathrm{KOH}$ (125 mg, $2.2 \mathrm{mmol})$, TBAB (44 mg, $0.13 \mathrm{mmol}$ ) in dry acetonitrile $(5 \mathrm{ml})$, during the addition the solution became yellow. The reaction mixture was stirred at $0{ }^{\circ} \mathrm{C}$ for $3 \mathrm{~h}$, then, filtered through glass cintered crucible to remove polymeric material. The filtrate was concentrated under reduced pressure and extracted with diethyl ether $(25 \mathrm{ml} \times 3)$, washed with water $(20 \mathrm{ml})$, brine $(20 \mathrm{ml})$, dried over $\mathrm{MgSO}_{4}$ and evaporated. The residue $(170 \mathrm{mg})$ was directly hydrolyzed with $\mathrm{HCl}$ in the next step.

The crude residue $(170 \mathrm{mg})$ was dissolved in diethyl ether $(5 \mathrm{ml})$ and was added $1 \mathrm{~N} \mathrm{HCl}(2$ $\mathrm{ml}$ ). After $4 \mathrm{~h}$ at RT, ether layer was separated and discarded. The aqueous layer was washed with diethyl ether to remove unwanted organic residues. Then, the aqueous layer was brought to $\mathrm{pH} \sim 10$ by adding ammonia solution, extracted with ethyl acetate $(25 \mathrm{ml} \times 3)$, washed with water $(25 \mathrm{ml})$, brine $(20 \mathrm{ml})$, dried over $\mathrm{MgSO}_{4}$ and evaporated. The residue gave amino ester $(57 \mathrm{mg})$ which was dissolve in dry dichloromethane $(5 \mathrm{ml})$ and were added a pinch of DMAP and acetic anhydride (55 mg, $0.54 \mathrm{mmol})$. After $12 \mathrm{~h}$ at $\mathrm{RT}, 50 \% \mathrm{NaHCO}_{3}$ solution $(4 \mathrm{ml})$ was added, extracted with ethyl acetate $(25 \mathrm{ml} \times 3)$, washed with water $(20 \mathrm{ml})$, brine $(20 \mathrm{ml})$, dried over $\mathrm{MgSO}_{4}$ and evaporated. The residue was chromatographed on silica gel column in $32 \%$ ethyl acetate/petroleum ether to lead to compound $8(18 \mathrm{mg}, 8 \%)$ as a semi solid. $R_{f}$ (ethyl acetate : petroleum ether, 1:1): 0.70; IR (neat) $v_{\max }$ : $3401(\mathrm{NH}), 1742$ (ester $\left.\mathrm{C}=\mathrm{O}\right), 1662\left(\mathrm{OCOCH}_{3}\right) \mathrm{cm}^{-1}$; ${ }^{1} \mathrm{H}$ NMR (300 MHz, $\mathrm{CDCl}_{3}$ ): $\delta 1.25\left(\mathrm{t}, \mathrm{J}=6.9 \mathrm{~Hz}, 3 \mathrm{H}\right.$, ester $\mathrm{CH}_{3}$ ), 1.99 (s, 3H, OCOMe), 2.09 (s, $3 \mathrm{H}, \mathrm{NHCOMe}$ ), 2.88 (s, 4H, $\mathrm{CH}_{2} \mathrm{CH}_{2}$ ), 3.08-3.11 (m, 2H, $\left.\mathrm{CH}_{2} \mathrm{CH}\right), 4.17$ (q. J=7.1 Hz, 2H, ester $\mathrm{OCH}_{2}$ ), 4.81-4.88 (m, 1H, $\left.\mathrm{CH}_{2} \mathrm{CH}\right), 5.07$ (s, 2H, $\left.\mathrm{CH}_{2} \mathrm{OAc}\right), 5.88$ (d, J=7.6 Hz, 1H, NH), 7.027.29 (m, 8H, Ar-H); Mass: m/z $411\left(\mathrm{M}^{+}\right)$; HRMS: m/z (EI) for $\mathrm{C}_{24} \mathrm{H}_{29} \mathrm{NO}_{5}$ Calcd: $(\mathrm{M}+\mathrm{H})$ 412.2124; Found: 412.2132.

Further eluted of the column with $80 \%$ ethyl acetate/petroleum ether for obtaining compound 9 (20 mg, 7\%) as a white solid. Mp: $168-170{ }^{\circ} \mathrm{C} ; R_{f}$ (ethyl acetate : petroleum ether, 1:1): 0.46; $\mathrm{UV}\left(\mathrm{CHCl}_{3}\right): \lambda_{\max }\left(\varepsilon \mathrm{M}^{-1} \mathrm{~cm}^{-1}\right) 257.5\left(1.25 \times 10^{3}\right) \mathrm{nm}$; IR $(\mathrm{KBr}) v_{\max }: 3255(\mathrm{NH}), 1749($ ester 
$\mathrm{C}=\mathrm{O}), 1644(\mathrm{NHC}=\mathrm{O}) \mathrm{cm}^{-1} ;{ }^{1} \mathrm{H}$ NMR $\left(300 \mathrm{MHz} \mathrm{CDCl}_{3}\right): \delta 1.25\left(\mathrm{t}, \mathrm{J}=7.1 \mathrm{~Hz}, 6 \mathrm{H}\right.$, ester $\left.\mathrm{CH}_{3}\right)$, $1.99\left(\mathrm{~s}, 6 \mathrm{H}, \mathrm{COCH}_{3}\right), 2.86\left(\mathrm{~s}, 4 \mathrm{H}, \mathrm{CH}_{2} \mathrm{CH}_{2}\right), 3.03-3.15$ (m, 4H, $\left.\mathrm{CH}_{2} \mathrm{CH}\right), 4.17$ (q. J=6.9 Hz, 4H, ester $\left.\mathrm{OCH}_{2}\right), 4.81-4.89\left(\mathrm{~m}, 2 \mathrm{H}, \mathrm{CH}_{2} \mathrm{CH}\right), 5.91(\mathrm{~d}, \mathrm{~J}=7.7 \mathrm{~Hz}, 1 \mathrm{H}, \mathrm{NH}), 6.04(\mathrm{~d}, \mathrm{~J}=7.5 \mathrm{~Hz}, 1 \mathrm{H}$, $\mathrm{NH}), 6.98-7.09(\mathrm{~m}, 8 \mathrm{H}, \mathrm{Ar}-\mathrm{H})$.; ${ }^{1} \mathrm{H}$ NMR (300 MHz, DMSO d $\left.\mathrm{d}_{6}\right): \delta 1.09(\mathrm{t}, \mathrm{J}=7.1 \mathrm{~Hz}, 6 \mathrm{H}$, ester $\left.\mathrm{CH}_{3}\right), 2.50\left(\mathrm{~s}, 6 \mathrm{H}, \mathrm{COCH}_{3}\right), 2.80\left(\mathrm{~s}, 4 \mathrm{H}, \mathrm{CH}_{2} \mathrm{CH}_{2}\right), 2.83-2.96\left(\mathrm{~m}, 4 \mathrm{H}, \mathrm{CH}_{2} \mathrm{CH}\right), 4.02$ (q. J=6.9 $\mathrm{Hz}, 4 \mathrm{H}$, ester $\left.\mathrm{OCH}_{2}\right), 4.34-4.41\left(\mathrm{~m}, 2 \mathrm{H}, \mathrm{CH}_{2} \mathrm{CH}\right), 7.11(\mathrm{~s}, 8 \mathrm{H}, \mathrm{Ar}-\mathrm{H}), 8.28$ (d, J=7.5 Hz, 2H, $\mathrm{NH}) ;{ }^{13} \mathrm{C}$ NMR (75.4 MHz, $\left.\mathrm{CDCl}_{3}\right): \delta$ 14.2, 23.2, 37.5, 37.6, 53.2, 61.5, 128.6, 129.3, 133.5, 140.5, 169.6, 171.7; Mass: m/z $496\left(\mathrm{M}^{+}\right)$; HRMS: m/z (EI) for $\mathrm{C}_{28} \mathrm{H}_{36} \mathrm{~N}_{2} \mathrm{O}_{6}$ Calcd: $(\mathrm{M}+\mathrm{H})$ 497.2652; Found 497.2659.

Synthesis of compound (8) and (9) in presence of miceller conditions. To a solution of N(diphenylmethylene)glycine ethyl ester (Schiff-base) (725 mg, $2.7 \mathrm{mmol}$ ), KOH (380 mg, 6.78 $\mathrm{mmol})$ and CTAB (54 mg, $0.14 \mathrm{mmol})$ in dry acetonitrile $(12 \mathrm{ml})$ was added drop wise a solution of dibromide $4(200 \mathrm{mg}, 0.54 \mathrm{mmol})$ in dry acetonitrile $(5 \mathrm{ml})$ at $0{ }^{\circ} \mathrm{C}$. After $1 \mathrm{~h}$ at RT solvent was removed, extracted with diethyl ether $(25 \mathrm{ml} \times 3)$, washed with water $(20 \mathrm{ml})$, brine $(20 \mathrm{ml})$, dried over $\mathrm{MgSO}_{4}$ and evaporated. The residue $(380 \mathrm{mg}$ ) was directly hydrolyzed with dilute $\mathrm{HCl}$ in the next step.

The crude residue $(380 \mathrm{mg})$ was dissolved in diethyl ether $(8 \mathrm{ml})$ and was added $1 \mathrm{~N} \mathrm{HCl} \mathrm{(2}$ $\mathrm{ml})$. After $4 \mathrm{~h}$ at RT ether layer was separated and discarded. The aqueous layer was washed with diethyl ether to remove organic impurities. Then, the aqueous layer was brought to $\mathrm{pH} \sim 10$ by adding ammonia solution, extracted with ethyl acetate $(25 \mathrm{ml} \times 3)$, washed with water $(25$ $\mathrm{ml})$, brine $(20 \mathrm{ml})$, dried over $\mathrm{MgSO}_{4}$ and evaporated. The residue gave amino ester (75 $\mathrm{mg}$ ) which was dissolve in dry dichloromethane $(5 \mathrm{ml})$ and were added a pinch of DMAP, and acetic anhydride $(65 \mathrm{mg}, 0.63 \mathrm{mmol})$. After $12 \mathrm{~h}$ at $\mathrm{RT}, 50 \% \mathrm{NaHCO}_{3}$ solution $(15 \mathrm{ml})$ was added, extracted with ethyl acetate $(25 \mathrm{ml} \times 3)$, washed with water $(20 \mathrm{ml})$, brine $(20 \mathrm{ml})$, dried over $\mathrm{MgSO}_{4}$ and evaporated The residue was chromatographed on silica gel in $32 \%$ ethyl acetate/petroleum ether to lead to compound $8(13.5 \mathrm{mg}, 6 \%)$ as a semi solid. Further eluted of the column with $80 \%$ ethyl acetate/petroleum ether for obtaining compound 9 (35 $\mathrm{mg}, 13 \%)$ as a white solid. The spectral data of $\mathbf{8}$ and $\mathbf{9}$ has found to be the same as described in the earlier experiment.

Synthesis of cyclophane derivative 10 under PTC conditions. To a solution of dibromide 4 (368 $\mathrm{mg}, 1 \mathrm{mmol})$, ethyl isocyanoacetate $(113 \mathrm{mg}, 1 \mathrm{mmol})$, TBAHS (tetrabutylammonium hydrogen sulfate) $(102 \mathrm{mg}, 0.3 \mathrm{mmol})$ in dry acetonitrile $(20 \mathrm{ml})$ was added finely powdered potassium carbonate $(830 \mathrm{mg}, 6.0 \mathrm{mmol})$ and the resulting heterogeneous mixture was refluxed at $80{ }^{\circ} \mathrm{C}$ for $12 \mathrm{~h}$. Then, the reaction mixture was cooled and filtered to remove the unwanted salts. The filtrate was concentrated under reduced pressure and the residue was taken in diethyl ether $(25 \mathrm{ml} \times 3)$, washed with water $(20 \mathrm{ml})$, brine $(15 \mathrm{ml})$, dried over $\mathrm{MgSO}_{4}$ and evaporated The residue was chromatographed on silica gel in 6\% ethyl acetate/petroleum ether to lead to product 10 (white solid) $(11.2 \mathrm{mg}, 3.5 \%)$ as a mixture of two isomers. Mp: $190-192{ }^{\circ} \mathrm{C} ; R_{f}$ (ethyl acetate : petroleum ether, 1:6): 0.47; $\mathrm{UV}\left(\mathrm{CHCl}_{3}\right): \lambda_{\max }\left(\varepsilon \mathrm{M}^{-1} \mathrm{~cm}^{-1}\right) 263.5\left(1.12 \times 10^{3}\right) \mathrm{nm}$; IR (KBr) $v_{\max }: 2140(\mathrm{NC}), 1749($ ester $\mathrm{C}=\mathrm{O}) \mathrm{cm}^{-1}$; ${ }^{1} \mathrm{H}$ NMR $\left(300 \mathrm{MHz}, \mathrm{CDCl}_{3}\right): \delta 1.18-1.28(\mathrm{~m}$, 
6H, ester $\left.\mathrm{CH}_{3}\right), 2.65-3.08\left(\mathrm{~m}, 16 \mathrm{H}, \mathrm{CH}_{2}\right), 4.14-4.24\left(\mathrm{~m}, 4 \mathrm{H}\right.$, ester $\left.\mathrm{OCH}_{2}\right), 6.72-6.92(\mathrm{~m}, 16 \mathrm{H}$, Ar-H). ${ }^{13} \mathrm{C}$ NMR $\left(75.4 \mathrm{MHz}, \mathrm{CDCl}_{3}\right): \delta 14.1,37.1,37.3,42.9,43.1,62.7,62.8,69.5,129.1$, 130.1, 130.5, 130.8, 140.0, 140.2, 160.5, 161.0, 168.4, 168.9; HRMS: m/z (EI) for $\mathrm{C}_{42} \mathrm{H}_{42} \mathrm{~N}_{2} \mathrm{O}_{4}$ Calcd: 638.3144; Found: 638.3115.

Synthesis of cyclophane derivative 10 in presence of BEMP conditions. To a stirred solution of dibromide $4(130 \mathrm{mg}, 0.35 \mathrm{mmol})$ and ethyl isocyanoacetate $(40 \mathrm{mg}, 0.35 \mathrm{mmol})$ in dry acetonitrile $(3 \mathrm{ml})$ was added drop wise BEMP $(193 \mathrm{mg}, 0.7 \mathrm{mmol})$ in dry actonitrile $(1 \mathrm{ml})$ at $0{ }^{\circ} \mathrm{C}$ under nitrogen. After 30 minutes at RT solvent was removed extracted with diethyl ether $(20 \mathrm{ml} \times$ $3)$, washed with water $(20 \mathrm{ml})$, brine $(20 \mathrm{ml})$, dried over $\mathrm{MgSO}_{4}$ and evaporated. The residue was chromatographed on silica gel in 5\% ethyl acetate/petroleum ether to lead to coupling product 10 (white solid) (16 mg, 14\%) as a mixture of two isomers.

Hydrolysis of $\mathbf{1 0}$ in presence of diethyl ether. To a stirred solution of the coupling product $\mathbf{1 0}$ (22.5 $\mathrm{mg}, 0.035 \mathrm{mmol}$ ) in purified diethyl ether $(8 \mathrm{ml})$ (diethyl ether was purified by passing through neutral alumina and then twice distilled over sodium wire to remove hydroquinone impurities) was added concd $\mathrm{HCl}(3$ drops $)$ at $0{ }^{\circ} \mathrm{C}$. After $7 \mathrm{~h}$ at $\mathrm{RT}$, diethyl ether $(50 \mathrm{ml})$ was added, washed with water $(15 \mathrm{ml})$, brine $(15 \mathrm{ml})$, dried over $\mathrm{MgSO}_{4}$ and evaporated. The residue was chromatographed on silica gel in $10 \%$ ethyl acetate/petroleum ether to lead to product 12 (11.5 mg, 48\%) as a white solid. Mp: $210{ }^{\circ} \mathrm{C}$ (decomp); . $R_{f}$ (ethyl acetate : petroleum ether, 3:7): 0.70; UV $\left(\mathrm{CHCl}_{3}\right): \lambda_{\max }\left(\varepsilon \mathrm{M}^{-1} \mathrm{~cm}^{-1}\right) 264.5\left(1.28 \times 10^{3}\right) \mathrm{nm}$; IR $(\mathrm{KBr}) v_{\max }: 3364(\mathrm{NH}), 1737$ $($ ester $\mathrm{C}=\mathrm{O}), 1680(\mathrm{NHC}=\mathrm{O}) \mathrm{cm}^{-1}$; ${ }^{1} \mathrm{H}$ NMR $\left(300 \mathrm{MHz}, \mathrm{CDCl}_{3}\right): \delta 1.43(\mathrm{t}, \mathrm{J}=7.1 \mathrm{~Hz}, 6 \mathrm{H}$, ester $\left.\mathrm{CH}_{3}\right), 3.01$ (s, 8H, $\left.\mathrm{CH}_{2} \mathrm{CH}_{2}\right), 3.05(1 / 2 \mathrm{ABq}, \mathrm{J}=13.3 \mathrm{~Hz}, 4 \mathrm{H}$, diastereotopic-H), 3.71 (1/2 ABq, $\mathrm{J}=13.3 \mathrm{~Hz}, 4 \mathrm{H}$, diastereotopic-H), 4.07 (q, J=7.1 Hz, 4H, ester $\left.\mathrm{OCH}_{2}\right), 6.71$ (d, J=8.0 Hz, 8H, Ar$\mathrm{H}), 6.76$ (br s, 2H, NH), 6.84 (d, J=8.0 Hz, 8H, Ar-H), 7.85 (d, J=1.8 Hz, 2H, CHO); ${ }^{13} \mathrm{C}$ NMR $\left(75.4 \mathrm{MHz}, \mathrm{CDCl}_{3}\right): \delta 14.2,34.7,40.0,62.0,66.7,128.5,129.3,133.2,138.9,160.7,171.8$; FAB Mass: m/z $675(\mathrm{M}+1)$; HRMS: m/z (positive ion FAB) for $\mathrm{C}_{42} \mathrm{H}_{46} \mathrm{~N}_{2} \mathrm{O}_{6}$ Calcd: (M+) 674.3354; Found: 674.3422.

Further eluted of the column with $30 \%$ ethyl acetate/petroleum for obtaining compound 11 (9.5 mg, 40\%) as a white solid. Mp: $220{ }^{\circ} \mathrm{C}$ (decomp); . $R_{f}$ (ethyl acetate : petroleum ether, 3:7): 0.30; UV $\left(\mathrm{CHCl}_{3}\right): \lambda_{\max }\left(\varepsilon \mathrm{M}^{-1} \mathrm{~cm}^{-1}\right) 265.0\left(1.07 \times 10^{3}\right) \mathrm{nm}$; IR $(\mathrm{KBr}) v_{\max }: 3389(\mathrm{NH}), 1737$ $($ ester $\mathrm{C}=\mathrm{O}), 1686(\mathrm{NHC}=\mathrm{O}) \mathrm{cm}^{-1} ;{ }^{1} \mathrm{H}$ NMR $\left(300 \mathrm{MHz}, \mathrm{CDCl}_{3}\right): \delta 1.28(\mathrm{t}, \mathrm{J}=7.3 \mathrm{~Hz}, 6 \mathrm{H}$, ester $\left.\mathrm{CH}_{3}\right), 2.97$ (s, 8H, $\left.\mathrm{CH}_{2} \mathrm{CH}_{2}\right), 3.16(1 / 2 \mathrm{ABq}, \mathrm{J}=13.5 \mathrm{~Hz}, 4 \mathrm{H}$, diastereotopic-H), 3.51 (1/2 ABq, $\mathrm{J}=13.5 \mathrm{~Hz}, 4 \mathrm{H}$, diastereotopic-H), 4.05 (q, $\mathrm{J}=7.3 \mathrm{~Hz}, 4 \mathrm{H}$, ester $\left.\mathrm{OCH}_{2}\right), 5.90(\mathrm{~s}, 2 \mathrm{H}, \mathrm{NH}), 6.71$ $6.83(\mathrm{~m}, 16 \mathrm{H}, \mathrm{Ar}-\mathrm{H}), 7.82(\mathrm{~d}, \mathrm{~J}=1.8 \mathrm{~Hz}, 2 \mathrm{H}, \mathrm{CHO}) ;{ }^{13} \mathrm{C} \mathrm{NMR}\left(75.4 \mathrm{MHz}, \mathrm{CDCl}_{3}\right): \delta 14.2,34.0$, $39.8,62.0,68.0,128.4,128.9,133.0,138.6,161.5,172.3 ; \mathrm{HRMS}: \mathrm{m} / \mathrm{z}$ (positive ion FAB) for $\mathrm{C}_{42} \mathrm{H}_{46} \mathrm{~N}_{2} \mathrm{O}_{6}$ Calcd: $(\mathrm{M}+\mathrm{H})$ 675.3434; Found: 675.3410 .

Synthesis of compound (15). ${ }^{17}$ A solution of 1,2 dibromo benzene $(200 \mathrm{mg}, 0.85 \mathrm{mmol}), p$ methyl phenylboronic acid (559 mg, $4.11 \mathrm{mmol}), \mathrm{Na}_{2} \mathrm{CO}_{3}(436 \mathrm{mg}, 4.11 \mathrm{mmol})$ in THF (10 ml), toluene $(10 \mathrm{ml})$, and water $(5 \mathrm{ml})$ were degased for $30 \mathrm{~min}$, then, $\mathrm{Pd}\left(\mathrm{PPh}_{3}\right)_{4}$ catalyst $(125 \mathrm{mg}$, $0.10 \mathrm{mmol}$ ) was added. The reaction mixture was heated at $80-90{ }^{\circ} \mathrm{C}$ for $30 \mathrm{~h}$, extracted with diethyl ether $(30 \mathrm{ml} \times 3)$, washed with water $(25 \mathrm{ml})$, brine $(25 \mathrm{ml})$, dried over $\mathrm{MgSO}_{4}$ and 
evaporated. The residue was chromatographed on silica gel in petroleum ether to lead to product 15 (188 mg, 86\%) as a white solid. Mp: $96-97{ }^{\circ} \mathrm{C} ; R_{f}$ (petroleum ether): $0.83 ;{ }^{1} \mathrm{H} \mathrm{NMR} \mathrm{(60} \mathrm{MHz,}$ $\left.\mathrm{CDCl}_{3}\right): \delta 2.3\left(\mathrm{~s}, 6 \mathrm{H}, \mathrm{CH}_{3}\right), 7.2(\mathrm{~s}, 8 \mathrm{H}, \mathrm{Ar}-\mathrm{H}), 7.4(\mathrm{~s}, 4 \mathrm{H}, \mathrm{Ar}-\mathrm{H})$.

Synthesis of cyclophane 16 in presence of BEMP. To a solution of dibromide 13 (100 mg, 0.24 $\mathrm{mmol})$, ethyl isocyanoacetate $(28 \mathrm{mg}, 0.24 \mathrm{mmol})$ in dry acetonitrile $(5 \mathrm{ml})$ was added drop-wise BEMP (150 mg, $0.54 \mathrm{mmol})$ solution in dry acetonitrile $(1 \mathrm{ml})$ at $0{ }^{\circ} \mathrm{C}$. After $20 \mathrm{~h}$ at RT, solvent was removed, extracted with diethyl ether $(20 \mathrm{ml} \times 3)$, washed with water $(20 \mathrm{ml})$, brine $(20 \mathrm{ml})$, dried over $\mathrm{MgSO}_{4}$. and evaporated. The residue was chromatographed on silica gel in $5 \%$ ethyl acetate/petroleum ether to lead to alkylated product $16(22 \mathrm{mg}, 25 \%)$ as a white solid. Mp: $245{ }^{\circ} \mathrm{C}$ (decomp); . $R_{f}$ (ethyl acetate : petroleum ether, 1:6): $0.38 ; \mathrm{UV}\left(\mathrm{CHCl}_{3}\right): \lambda_{\max }\left(\varepsilon \mathrm{M}^{-1} \mathrm{~cm}^{-1}\right)$ $246.0\left(4.39 \times 10^{4}\right) \mathrm{nm}$; IR $(\mathrm{KBr}) v_{\max }: 2141(\mathrm{NC}), 1750($ ester $\mathrm{C}=\mathrm{O}) \mathrm{cm}^{-1} ;{ }^{1} \mathrm{H}$ NMR $(300 \mathrm{MHz}$, $\left.\mathrm{CDCl}_{3}\right): \delta$ 1.24-1.29 (m, 6H, ester $\left.\mathrm{CH}_{3}\right), 2.74-3.15\left(\mathrm{~m}, 8 \mathrm{H}, \mathrm{CH}_{2}\right), 4.21-4.29\left(\mathrm{~m}, 4 \mathrm{H}\right.$, ester $\left.\mathrm{OCH}_{2}\right)$, 7.03 (d, J=1.8 Hz, $16 \mathrm{H}$, Ar-H), 7.43 (d, J=3.3 Hz, 8H, Ar-H). FAB mass: m/z 735 (M+1); HRMS: m/z (positive ion FAB) for $\mathrm{C}_{50} \mathrm{H}_{42} \mathrm{~N}_{2} \mathrm{O}_{4}$ Calcd: $(\mathrm{M}+\mathrm{H})$ 735.3222; Found: 735.3210 .

Hydrolysis of compound 16. To a stirred solution of isonitrile derivative 16 (20 $\mathrm{mg}, 0.027$ $\mathrm{mmol})$ in diethyl ether $(8 \mathrm{ml})$ and dichloromethane $(2 \mathrm{ml})$ was added concd $\mathrm{HCl}(2$ drops $)$ at $0{ }^{\circ} \mathrm{C}$. After $1 \mathrm{~h}$ at RT, solvent was removed, extracted with diethyl ether $(20 \mathrm{ml} \times 3)$, washed with water $(20 \mathrm{ml})$, brine $(15 \mathrm{ml})$, dried over $\mathrm{MgSO}_{4}$ and evaporated. The residue was chromatographed on silica gel in $16 \%$ ethyl acetate/petroleum ether to lead to $\mathrm{N}$-formyl derivative $17(13 \mathrm{mg}, 62 \%)$ as a mixture of isomers. Mp: $230^{\circ} \mathrm{C}$ (decomp); . $R_{f}$ (ethyl acetate : petroleum ether, 3:7): 0.42; UV $\left(\mathrm{CHCl}_{3}\right): \lambda_{\max }\left(\varepsilon \mathrm{M}^{-1} \mathrm{~cm}^{-1}\right) 246.0\left(3.85 \times 10^{4}\right) \mathrm{nm}$; IR $(\mathrm{KBr}) v_{\max }$ : $3371(\mathrm{NH}), 1736($ ester $\mathrm{C}=\mathrm{O}), 1675(\mathrm{NHC}=\mathrm{O}) \mathrm{cm}^{-1} ;{ }^{1} \mathrm{H} \mathrm{NMR}\left(300 \mathrm{MHz}, \mathrm{CDCl}_{3}\right): \delta 1.33(\mathrm{t}$, $\mathrm{J}=7.3 \mathrm{~Hz}, 6 \mathrm{H}$, ester $\mathrm{CH}_{3}$ ), 3.00-3.88 (m, 8H, $\mathrm{CH}_{2}$ ), 4.3 (q, J=7.3 Hz, 4H, ester $\left.\mathrm{OCH}_{2}\right), 6.12$ (s, 2H, NH), 6.83-6.97 (m, 8H, Ar-H), 7.27-7.94 (m, 16H, Ar-H), 7.94 (d, J=1.8 Hz, 1H, CHO), $8.02\left(\mathrm{~d}, \mathrm{~J}=1.5 \mathrm{~Hz}, 1 \mathrm{H}, \mathrm{CHO}\right.$ ); HRMS: $\mathrm{m} / \mathrm{z}$ (positive ion $\mathrm{FAB}$ ) for $\mathrm{C}_{50} \mathrm{H}_{46} \mathrm{~N}_{2} \mathrm{O}_{6} \mathrm{Calcd}$ : $(\mathrm{M}+\mathrm{H}$ ) 771.3434; Found: 771.3424.

\section{Acknowledgements}

We gratefully acknowledge the NST for the financial support, RSIC Mumbai and TIFR for recording the spectral data. S. H. thanks IIT-Bombay for the award of research fellowship.

\section{References}

1. (a) Balaram, P. Curr. Opin. Struct. Biol. 1992, 2, 845. (b) Toniolo, C.; Crisma, M.; Fabiano, N.; Melchiorri, P.; Negri, L.; Krause, J. A.; Eggfeston, D. S. Int. J. Peptide Proein Res. 1994, 44, 85. (c) Wysong, C. L.; Yokum, T. S.; McLaughlin, M. L.; Hammer, R. P. Chemtech. 1997, 26. (d) Kaul, R.; Balaram, P. Bioorg. Med. Chem. 1999, 7, 105. (e) 
Cativiela, C.; Diaz-de-Villegas, M. D. Tetrahedron: Asymmetry 2000, 11, 645. (f) Gibson, S. E.; Guillo, N.; Tozer, M. J. Tetrahedron 1999, 55, 585. (g) Giannis, A.; Kolter, T. Angew. Chem., Int. Ed. 1993, 32, 1244. (h) Duthaler, R. O. Tetrahedron 1994, 50, 1539.

2. (a) Keehn, P. M.; Rosenfeld, S. M. Cyclophanes; Vol. 1 \& 2 Academic Press: New York, 1983 and references cited therein. (b) Lehn, J-M. Supramolecular Chemistry: Concept and Perspective; VCH: Weinheim, 1995. (c) Cram, D. J. From Design to Discovery; Washington, DC, 1990.

3. Pelter, A.; Crump, R. A. N. C.; Kidwell, H. Tetrahedron Lett. 1996, 37, 127. (b) Pelter, A.; Kidwell, H.; Crump, R. A. N. C. Chem. Soc., Perkin Trans. I 1997, 3137. (c) Marchand, A.; Maxwell, A.; Mootoo, B.; Pelter, A.; Reid, A. Tetrahedron 2000, 56, 7331.

4. Kotha, S.; Sreenivasachary, N. Indian. J. Chem. 2001, 40B, 763. (b) Trnka, T. M.; Grubbs, R. H. Acc. Chem. Res. 2001, 34, 18. (c) Hoveyda, A. H.; Schrock, R. R. Chem. Eur. J. 2001, 945.

5. Sasaki, H.; Kitagawa, T. Chem. Pharm. Bull. 1983, 31, 2868.

6. (a) Kotha, S.; Brahmachary, E. J. Org. Chem. 2000, 65, 1359. (b) Kotha, S. Acc. Chem. Res. 2003, 36, 342.

7. Kotha, S.; Halder, S.; Damodharan, L.; Pattabhi, V. Bioorg. Med. Chem Lett. 2002, 12, 1113.

8. O’Donnell, M. J.; Polt, R. L. J. Org. Chem. 1982, 47, 2663.

9. Cram, D. J.; Steinberg, H. J. Am. Chem. Soc. 1951, 73, 5691.

10. Okino, T.; Takemoto, Y. Org. Lett. 2001, 3, 1515.

11. Basu, B.; Chattopadhyay, S. K.; Ritzen, A.; Frejd, T. Tetrahedron: Asymmetry 1997, 8, 1841. (b) Lygo, B.; Crosby, J.; Peterson, J. A.; Tetrahedron 2001, 57, 6447.

12. Miranda, M. A.; Font-Sanchis, E.; Perez-Prieto, J.; Scaiano, J. C. J. Org. Chem. 2001, 66, 2717.

13. Schwesinger, R. Chimia 1985, 39, 269. (b) Schwesinger, R. Nachr. Chem. Tech. Lab. 1990, 38, 1214. (c) Schwesinger, R.; Willaredt, J.; Schlemper, H.; Keller, M.; Schimitt, D.; Fritz, H. Chem. Ber. 1994, 127, 2435.

14. Damodharan, L.; Ibrahim, B. S. Pattabhi, V.; Halder, S.; Kotha, S. Acta Cryst. 2002, E58, 1038.

15. (a) Kotha, S.; Lahiri, K.; Kashinath, D. Tetrahedron 2002, 58, 9633. (b) Kotha, S.; Lahiri, K. Biopolymers 2003, 69517.

16. Chan, C. W.; Wong, H. N. C. J. Am. Chem. Soc. 1988, 110, 462. Hammerschmidt, E.; Vögtle, F. Chem. Ber. 1980, 113, 1125.

17. (a) Roth, W. R.; Unger, C; Wasser, T. Liebigs Ann. 1996, 12, 2155. (b) Jacobson, N; Boekelheide, V. Angew. Chem., Int. Ed. 1978, 17, 46. 